\title{
Ruptures des liens familiaux et processus de socialisation de l'enfant
}

\author{
Jorge Alberto Serrano \\ Université Catholique de Louvain \\ Verónica Serrano \\ Universidad Mayor de San Simón
}

\begin{abstract}
Résumé
Les auteurs rappèlent l'importance du contexte socio-familial dans le développement de l'enfant, puisque l'absence d'une structure familiale suffisamment contenante, fondée sur la constitution des liens d'attachement suffisamment solides, peut entraîner une certaine vulnérabilité dans le développement des fonctions de socialisation. L'affaiblissement ou les "ruptures" des liens familiaux peuvent ainsi favoriser l'émergence de certaines difficultés psychopathologiques chez l'enfant. Les modalités d'attachement précoce de l'enfant mises en rapport avec ces fonctions peuvent configurer des formes particulières, sinon spécifiques de comportement social. Les auteurs proposent un modèle croisé de ces deux groupe de facteurs que les intervenants doivent prendre en considération pour entreprendre des mesures thérapeutiques avec ces enfants en "rupture" des liens familiaux.
\end{abstract}

Mots-clés: Attachement; rupture des liens familiaux; socialisation de l'enfant.

\begin{abstract}
Family relationship breakdown and child socialisation

The authors remind the importance of the socio-familiar context in the child development, insisting that the lack of a family structure enough containing, based on the establishment of consistent attachment binds, could favour some kind of child social functions vulnerability. The weakening or the breakdown of family relationships could facilitate the emergence of children psychopathological disturbances. The modalities of child early attachment, cross-over with the development of child social functions, allows the authors to describe some specific-like social behaviours, that must be considered working with those children confronted with family relationship breakdown.
\end{abstract}

Keywords: Attachment; family relationship breakdown; child socialisation.

\section{Introduction}

Le développement de l'enfant s'enracine dans l'établissement des liens affectifs solides et stables, en particulier, pendant les premières années de sa vie. Or, les conditions socioéconomiques, la mobilité géographique ainsi que le questionnement de certaines valeurs entraîné par la modernité, placent un nombre certain d'enfants dans des situations de rupture des liens familiaux.

Dans cet article, nous limiterons notre analyse à l'impact de l'affaiblissement ou des "ruptures" des liens familiaux sur les modalités d'attachement et les fonctions de socialisation de l'enfant, pouvant rendre ce dernier vulnérable à l'émergence des difficultés psychopathologiques ultérieures. Nous avons choisit à dessein le mot "ruptures" au pluriel et entre guillemets, pour ratisser large dans les conditions de vie de ces enfants.

En ce qui concerne plus précisément les situations de rupture, il faudrait établir une distinction entre des enfants abandonnés à leur naissance, - nés sous X, confiés en adoption ou "oubliés" dans les endroits les plus invraisemblables - parce que les parents ne savent pas ou ne veulent pas s'en occuper; et des enfants dont la rupture est plus tardive, des enfants orphelins coupés involontairement de leurs parents suite à des situations dramatiques comme les catastrophes naturelles ou les guerres, sans oublier les mineurs étrangers non accompagnés, souvent victimes d'organisations mafieuses qui les poussent à la prostitution ou au travail clandestin. Dans l'entre-deux se retrouvent, entre autres, les enfants de la rue ou dans la rue, les enfants soldats, ... très souvent livrés à eux-mêmes sans le support du milieu familial d'origine.

Cependant, toutes ces situations reflètent un certain affaiblissement du lien familial ainsi que des défaillances de la structure sociale qui ne parviennent pas à offrir à l'enfant, à tout enfant, la protection de la famille ou de la communauté (art. 5) et l'accès à un nom et à une nationalité (art. 7), comme l'impose la Convention Internationale des Droits de l'Enfant ${ }^{1}$.

\section{Contexte sociofamilial et développement de l'enfant}

Pourtant, ces liens sont indispensables pour assurer leur développement harmonieux tandis que les conséquences de leur absence sont imprévisibles. Ainsi, pour mieux cerner l'impact de la "rupture" familiale, nous suivrons trois fils conducteurs: 
$1^{\circ}$ ) Les enfants ou les adolescents abandonnés ou en rupture de liens, "perdent" leur ancrage familial ainsi que leurs repères psychosociaux (symboliques) indispensables pour leur développement.

$2^{\circ}$ ) Ces enfants courent courent le risque, à court et à long terme, de développer des liens d'attachement et de repères symboliques dysfonctionnels.

$3^{\circ}$ ) L'attachement et les repères psychosociaux sont des concepts étroitement liés et corrélatifs au développement psychosocial de toute personne.

Et pour illustrer certains de nos propos, nous aimerions présenter une vignette clinique.

Soufian, 10 ans, est orienté vers notre service de pédopsychiatrie par le juge des enfants qui demande un placement d'urgence.

Nous retrouvons un enfant au regard farouche, qui bouge en permanence pendant tout l'entretien. Il ne nous apprend pas grand-chose sur sa situation, puisque son intérêt et son discours, peu abondant, sont polarisés par son désir de rentrer chez lui. Toutefois nous parvenons à établir avec lui un bon contact, ce qui nous oriente sur la demande affective vécue par Soufian, dont l'agitation révèle cette quête affective.

L'assistante sociale qui l'accompagne nous apprend que la grand-mère maternelle, responsable de la garde de l'enfant, a demandé son placement parce qu'elle ne parvenait plus à s'en occuper convena-blement. Chez la grand-mère, Soufian se montre particulièrement difficile et exigeant; il n'admet pas des contraintes, traîne dans la rue des heures durant avec des jeunes plus âgés que lui; ses résultats scolaires évoluent en dents-de-scie. Dès lors, pour la grand-mère, elle-même souffrante et sans ressources, la situation devient ingérable.

L'assistante sociale nous apprend aussi que le père, toxicomane, n'a jamais donné de signes de vie. Il s'est suicidé par overdose. La mère, après un passé d'alcoolisme, de prostitution et de drogues, la rendant peu disponible pour son fils, a aussi mis fin à ses jours, quelques années auparavant.

Soufian est ainsi devenu un électron libre, soutenu vaille que vaille par sa grand-mère, elle-même limitée dans sa capacité de le "nourrir" et de le contenir affectivement pour lui proposer une structure familiale substitutive stable et structurante.

Après avoir négocié avec la grand-mère, Soufian quitte l'hôpital et retourne chez une tante qui, quelques jours après, ramène l'enfant avec une nouvelle procédure de placement, et alors, il est orienté vers un centre de psychiatrie infantile. À partir de ce moment s'initie une longue marche des intervenants psychosociaux pour reconstruire des liens de famille capables d'assurer au mieux son développement.

La situation de Soufian n'est, hélas!, pas unique, même si elle n'est pas non plus aussi dramatique dans tous les cas. Dans notre pratique clinique, nous avons rencontré des dizaines d'enfants pour lesquels l'intervention spécialisée est demandée suite à des manifestations psychopathologiques centrées essentiellement sur des troubles scolaires et du comportement. Ces plaintes sont souvent en relation avec leur vie quotidienne et touchent ce qui constitue la trame même de leur existence: leurs attachements, leur vie familiale. En effet, les enfants qui ne parviennent pas à s'intégrer correctement dans leurs structures familiales ou dans des institutions scolaires ou autres proviennent fréquemment des milieux familiaux partiellement dissociés ou inadaptés.

Les plaintes révèlent, nous semble-t-il, l'insuffisance, voire l'absence d'une structure familiale suffisamment contenante, apte à soutenir l'enfant dans son développement individuel et social. Le Rapport Annuel du Défenseur des Enfants de France, souligne par exemple, que leur souffrance fait suite à la rupture du lien social: "à l'éclatement de leur famille, à la séparation de ceux qu'ils aiment le plus, leurs parents, à la perte de repères qui en résulte, au sentiment d'arrachement qui consiste à ne plus voir l'un, ou l'autre".

Des lors, comment comprendre cette situation sans se référer au contexte socioéconomique et culturel actuel? La société européenne occidentale est traversée par des courants centrifuges de morcellement familiale ${ }^{2}$. Nul doute que ce fait se répercute sur la qualité des liens affectifs indispensables pour l'enfant, qui, par ailleurs, devient témoin impuissant de la violence des déchirements parentaux. À ce fait idéologiques'ajoutent la mobilité géographique, les exigences professionnelles qui pèsent sur les parents, la montée de la violence urbaine, le chômage qui creuse les inégalités, autant de conditions socioéconomiques qui renforcent l'affaiblissement de la cohésion sociale. Ces facteurs fragilisent l'enfant, même si ils n'interviennent que pour conditionner ou surdéterminer les situations d'abandon ou de rupture définitive des liens fami-liaux.

Cela revient à souligner l'importance du contexte social et culturel dans le processus d'humanisation de l'enfant. L'enfant ne se construit pas ailleurs que dans la rencontre avec les autres, les autres qui lui sont proches: famille "naturelle" ou substitutive. C'est parce que le sujet a besoin d'appartenir à un groupe qui l'accepte de manière inconditionnelle: le groupe familial, les pairs, l'école, le travail ou autre; il a aussi besoin de prendre sa vie en main et de faire preuve d'une certaine autonomie personnelle par rapport à son contexte; il a également besoin de se sentir reconnu dans les domaines qui lui sont propres et de préserver le sentiment de gérer son existence; et encore, le besoin de partager son temps et son bienêtre matériel avec autrui. L'inconsistance des liens émotionnels vécus par l'enfant rend hasardeuse la satisfaction de tous ces besoins. 
La place de l'enfant au sein d'une famille semble donc plus largement dépendante des valeurs symboliques d'une société donnée que des liens biologiques. C'est souvent le contexte socioculturel qui définit la fonction et la place de l'enfance dans le corps familial et sociétal.

\section{Structure familiale, attachement et psychopathologie infantile}

Pour mieux cerner l'importance de la structure familiale il faudrait s'entendre sur la notion de "famille" et évoquer d'autres conceptscorollaires tels que la "parentalité", la "filiation", "l'avenir de l'enfant en rupture du lien social", "les institutions substitutives" ... Impossible d' aborder toutes ces questions.

Tout enfant est issu de la rencontre, fortuite ou permanente, entre une femme et un homme, rencontre d'amour, rencontre de passion, rencontre de pulsion. Ce couple biologique, uni par le sexe et par la procréation, s'intègre naturellement dans une "famille" considérée comme l'ensemble organisé d'individus qui établissent entre eux des liens d'alliance ou de parenté. L'origine d'une famille s'établit sur cet enracinement biologique et temporel de la sexualité et de la procréation, mais reste largement ouverte au contexte socioculturel qui donne un sens à ce que l'on considère, depuis toujours, comme la "cellule de base de toute société".

Les faits de nature poussent les hommes et les femmes à s'unir et à procréer, mais ils sont irréductibles aux instincts animaux. Dès lors la famille ne peut, à son tour, être réduite à une réalité purement biologique. Les études anthropologiques attestent de l'importance des facteurs dits culturels dans les formes et les fonctions non biologiques de la famille. La filiation ne peut donc pas inscrire la parenté naturelle sur le seul fait biologique.

Le processus de subjectivation de l'enfant se développe grâce à sa relation à l'autre, à l'altérité, "aux paroles de papa et de maman", socle sur lequel il construit des valeurs normatives personnelles qui lui serviront de référence pour assumer sa responsabilité dans le monde ${ }^{3}$ et pour agir comme un sujet à son nom personnel, dans une perspective éthique, relatio-nnelle et créatrice. De ce point de vue, il importe de reprendre conscience du rôle fondateur des grands interdits, tel celui de l'inceste, pour la construction du sujet humain dans sa relation, instituée par le droit ou la coutume, avec les autres membres de sa famille ${ }^{4}$.

Malgré les difficultés, incertitudes et conflits rencontrés par le rôle fondateur des interdits, il s'agit pourtant de trouver un certain équilibre entre la liberté et les contraintes, entre les droits et les devoirs, autrement les fondements du lien social et de la fonction référentielle des liens familiaux seraient euxmêmes en danger.

La relation à l'autre, moulée par les interdits fondateurs, passe chez le jeune enfant par la constitution de l'attachement, au sein d'une structure sociale "familiale" naturelle ou substitutive, nucléaire ou élargie. L'attachement, faut-il le dire, est cette disposition puissante qui pousse l'enfant (l'individu) à chercher la proximité et le contact avec une figure spécifique, quand il a peur, quant il se sent fatigué ou malade. Cette figure d'attachement change peu au cours du temps, tout en s'inscrivant dans un système hiérarchique de préférences. C'est donc la rupture de ce lien assurant sa sécurité de base, suite à des séparations ou des pertes, qui provoque des perturbations dans la construction de la personnalité de l'enfant. Les enfants abandonnés vivent souvent plusieurs ruptures successives.

Le concept d'attachement a été contesté tant du point de vue théorique que méthodologique (Zeanah, 1996), même si l'existence de manifestations comportementales caractérisées par une approche sociale indiscriminée ainsi que par un manque de proximité sélective a été l'objet d'observa-tions cliniques classiques (Goldfarb, 1945; Provence et Lipton, 1962; Tizard et Rees, 1975), ainsi que plus récentes (Lieberman et Zeanah, 1995; Boris et al., 1998; Chisholm, 1998; O'Connor et al., 1999). Ces études ont montré la relation qui existe entre la négligence ou la déprivation et les troubles de l'attachement ainsi que la différence existante entre ces derniers et d'autres troubles dont l'origine serait plutôt d'ordre neuropsychologique. Pensons à la "personnalité dépourvue de tendresse" décrite par Bowbly $\left(1944^{4}-1969^{5}\right)$, qui prolonge les études de Spitz sur la carence maternelle.

D'autre part, la théorie de l'attachement, observée dans différents contextes s'occupant d'enfants (Rutter et O'Connor, 1999, O'Connor, 2000) a permis de développer aussi des procédures thérapeutiques adéquates (Lieberman, 1991).

Il y a donc un lien clair entre l'absence de structure familiale contenante et certains comportements sociaux à risque, dans la mesure ou les parents sont les agents prioritaires de la socialisation des enfants. Encore davantage cette absence entrave la constitution du surmoi, l'émergence de la culpabilité et, par conséquent, l'inhibition des pulsions.

Ainsi, les enfants issus des familles éclatées (divorces, séparation) ou monoparentales connaissent plus de difficultés d'intégration que ceux des familles unies ou des familles où l'un des parents est décédé. Que dire encore des enfants de la rue ou d'autres livrés à eux-mêmes, véritable enfance déboussolée, souvent aspirée par des bandes mafieuses qui développent ses tentacules dans nombre de agglomérations sur fond de crise économique.

Dès lors il convient de se demander comment agit la rupture des liens familiaux sur l'enfant, sur son processus de devenir sujet - sa subjectivation - et d'entrer en relation avec l'autre?

La fonction d'attachement serait une fonction adaptative de protection sociale primaire non réductible à la satisfaction des besoins physiologiques ou matériels. Elle s'exprime par des comportements d'attachement (contact visuel, sourire, pleurs, s'accrocher - coller - à quelqu'un...) souvent activés par la douleur, la fatigue ou les craintes. Ces 
comportements engagés par le sujet sont orientés en vue obtenir et/ou de maintenir la proximité avec la figure d'attachement. Le comportement de l'enfant (du sujet humain) trouve son pendant dans l'attitude parentale qui s'exprime par les moyens employés par les personnes proches de l'enfant pour répondre à ses demandes.

Selon Ainsworth (1967), l'interaction précoce avec les parents conditionne le type d'attachement que l'enfant développe: sécurisé, anxieux évitant et anxieux résistant ou ambivalent. L'enfant avec un "attachement sécurisé" est celui qui proteste en cas de séparation, recherche la proximité et montre un comportement de base sécurisant. L'enfant avec un attachement "anxieux évitant" est apparemment peu perturbé par la situation, faisant mine de ne pas être affecté par la séparation, ni d'avoir besoin de réconfort. Il se montre fort indépendant. L'enfant "anxieux, résistant ou ambivalent" se montre anxieux et parfois agité lors de la séparation. Il cherche le réconfort d'une manière ambivalente en s'accrochant d'abord a la figure d'attachement pour s'en défaire ensuite dans un mouvement de colère. À ces trois types, Main et al. (1985) ont ajouté une quatrième configuration l'attachement désorganisé, dont l'évolution est souvent problématique et entraîne des manifestations psychopathologiques comme les troubles du comportement. L'enfant, avec un attachement "désorganisé ou désorienté", apparaît désorienté par la séparation, montrant des signes d'appréhension, de confusion, de sidération ou de désorganisation; ses mouvements de rapprochement restent souvent incomplets et il se comporte comme étant plus alarmé par le parent lui-même que par la situation de séparation. Son comportement reflète le manque de repères venant du monde des adultes, qui ne peuvent pas accepter la vie émotionnelle de l'enfant, puisqu'ils ont été eux-mêmes marqués par un traumatisme vécu pendant leur enfance.

Ces différentes formes d'attachement contribuent à établir, souvent à long terme, des schémas de réaction à la séparation (cfr. Ainsworth, 1967), c'est-à-dire, des manières d'entrer en relation avec l'autre et de réagir à des situations de séparation.

Les perturbations des liens d'attachement (perte, inadéquation, déprivation) sont à la base de ce qu'on connaît actuellement sous le terme de troubles de l'attachement. La DSM IV et la DSM IV TR décrivent comme critères diagnostiques:

Un mode de relation sociale gravement perturbé et inapproprié au stade du développement, qui n'est pas imputable à un retard du développement. $\mathrm{Ce}$ mode de relation se manifeste, d'une part, par une incapacité persistante à engager des interactions sociales ou à y répondre d'une manière appropriée, et d'autre part, par l'existence de liens d'attachement diffus. Ce tableau correspond à une carence de soins adaptés à la suite de négligences à l'égard des besoins émotionnels ou physiques élémentaires de l'enfant, ainsi qu'à des changements fréquents des personnes s'en occupant.
Les troubles de l'attachement peuvent donc apparaître quand l'enfant a été abusé ou négligé, quand il a vécu dans des orphelinats pauvrement investis, ou quand il a changé fréquemment de milieu de vie. Cependant, tous les enfants adoptés, institutionnalisés ou menant leur existence dans la rue ne développeront nécessairement pas des troubles de l'attachement.

Comme l'ont montré Barbara et Jack Tizard (1972, 1977), l'évolution des bébés en institution n'est pas forcément dramatique et les effets de la séparation ne sont pas toujours irréversibles. C'est la présence de la mère ou de son substitut en tant que contenant des pulsions agressives de l'enfant qui est fondamentale; c'est-à-dire, la présence de l'adulte capable d'établir un contexte de base sécurisant qui permette à l'enfant de s'ouvrir au monde extérieur et d'accéder au monde symbolique.

Les troubles de l'attachement s'inscrivent dans un continuum entre des situations relativement légères et d'autres plus sévères de perte référentielle des liens familiaux. Entre des situations d'attachement "vulnérable" de l'enfant qui manque d'assurance et peut montrer des comportements "faussés" et l'attachement absent de l'enfant complètement "détaché", toutes les gammes comportementales sont possibles. Dès lors, nous avons préféré simplifier les différents types d'attachement les classant en trois catégories: sécurisé, perturbé (qui regroupe les formes d'attachement anxieux) et désorganisé (cfr. Tableau).

Les circonstances de la vie peuvent, bien entendu, modifier la qualité des attachements. Pensons par exemple à la trajectoire de Tim Guénard (1999) qui, après avoir vécu un passé institutionnel chargé, est parvenu à retrouver un sens à son existence. Pensons aussi à ces centaines de milliers d'enfants de la rue qui retrouvent dans les bandes d'enfants de banlieue les mécanismes identitaires et, en eux-mêmes, les ressources de résilience qui leur permet de dépasser les obstacles de la vie.

Ces enfants s'en sortent à la suite d'une rencontre, d'une expérience spirituelle, à la faveur d'un milieu les accueillant positivement et inconditionnellement ou grâce à la construction d'une "famille" imaginaire, à condition, pour cette dernière éventualité, qu'ils aient pu développer des capacités de symbolisation et de représentation suffisantes. En effet, dans un nombre important de situations d'abandon précoce l'enfant est plutôt coupé de sa capacité de représentation affective et développe ce que certains auteurs décrivent comme la "vie opératoire", caractérisée par un maniement rationnel des représentations coupées de ses racines imaginaires et affectives.

Les types d'attachement sont à mettre en rapport avec une série de fonctions (cfr. Pierce, 1998) que l'enfant développe pendant son processus de socialisation:

* Fonctions relationnelles et d'affiliation.

* Fonctions d'indépendance et d'autonomie.

* Fonctions de maîtrise et d'accomplissement personnel. 


\section{* Fonctions de générosité et d'altruisme.}

Sur le plan relationnel, les personnes qui ont développé des liens d'attachement sécurisé sont capables des relations d'amitié, de coopération, de respect, de confiance et d'amour relativement solides. Celles qui montrent des signes d'attachement anxieux perturbé (anxieux ou ambivalent) cherchent à attirer l'attention, sont en demande d'affection et d'approbation, se montrent collantes et changent souvent de partenaires affectifs. Finalement, les personnes qui ont des liens d'attachement désorganisés ou absents, ont tendance à s'isoler socialement, à se sentir rejetées, à se montrer méfiantes et à établir des relations affectives distantes ou superficielles.

En ce qui concerne l'autonomie, les personnes qui ont développé un attachement sécurisé se montrent confiantes, responsables, sûres d'elles-mêmes, assumant leur indépendance sereinement. Les personnes qui montrent des signes d'attachement perturbé (anxieux ou d'évitement) se montrent révoltées, téméraires, manipulatrices, passant de l'agressivité à la passivité facilement; elles sont affectivement instables et se sentent facilement discriminées, mises de côté. Finalement, l'attachement désorganisé se manifeste par l'apparente confiance en soi de celui qui camoufle un sentiment d'infériorité ou d'impuissance. Ces personnes sont dominées par un sentiment de désarroi, appris dès leur jeune âge, et ne parviennent pas à gérer convenablement leurs pulsions.
Pour ce qui est de la réalisation personnelle, les sujets qui ont établi des liens d'attachement sécurisés sont capables de faire face aux problèmes de leur existence, se montrent créatifs, "gagnants", résilients, motivés et "accrocheurs". L'attachement perturbé donne lieu à des comportements de travail incessant (workalcoholic) jamais suffisamment rassurant; enfin, les personnes qui ont développé un attachement désorganisé sont souvent en position de rivalité, de tricherie, de pratiques douteuses, prenant des risques dans leurs activités sociales et professionnelles.

Enfin, sur le plan de l'altruisme, les personnes sécurisées sont capables de compassion, d'empathie et de s'occuper d'autrui. L'attachement perturbé donne lieu à des attitudes égoïstes, d'implication excessive, de fausse soumission et dépendance. L'attachement absent ou désorganisé se traduit par des comportements narcissiques, hédonistes, anti-sociaux, voire psychopathiques (exploitation de l'autre).

Le tableau 1 reprend l'interaction entre les types d'attachement et les fonctions sociales. Ce sont surtout les enfants abandonnés ou vivant des ruptures familiales définitives plus tardives qui développent des formes d'attachement perturbé ou désorganisé. La vulnérabilité psychologique de ces enfants se traduit par l'émergence des difficultés psychopathologiques, et donc par la psychiatrisation ultérieure signalée au début de notre exposé. Quant aux enfants de parents divorcés, évoqués en filigrane, ils montrent à long terme, une certaine sensibilité à s'engager dans des relations stables.

\section{TYPES D'ATTACHEMENT}

\begin{tabular}{lll}
\hline SECURISÉ & ANXIEUX PERTURBÉ & DÉSORGANISÉ
\end{tabular}

\section{FONCTION SOCIALES}

$\begin{array}{ll}\begin{array}{l}\text { Relationnelles ou amitié, } \\ \text { d'affiliation }\end{array} & \begin{array}{l}\text { coopération, } \\ \text { respect, confiance, } \\ \text { amour }\end{array}\end{array}$

Indépendance et autonomie

Maîtrise et
accomplissement
personnel

Générosité et altruisme attirer l'attention, demander l'affection, "coller", changer de partenaires

révolte, témérité, manipulation, agressivité - passivité, instabilité affective

travail excessif, jamais rassurant, "workoholic" agitation, impulsivité

égoïsme, dépendance, fausse soumission, implication excessive

\author{
isolement social, \\ sentiment de rejet, \\ méfiance, \\ distance relationnelle \\ confiance apparente, \\ sentiment infériorité \\ et désarroi \\ faible gestion pulsions \\ rivalité, \\ tricherie, \\ pratiques douteuses \\ narcissisme, \\ hédonisme, \\ exploitation d'autrui
}

L'histoire d'un nombre plus ou moins important d'enfants est donc marquée par l'existence de liens précoces d'attachement dysfonctionnel. Cependant, pour qu'il y ait rupture, il faut que des liens d'attachement, même ténus, aient existé. D'autre part, 
les expériences ultérieures peuvent modifier l'impact de ces liens précoces d'attachement.

\section{Le rôle des intervenants psychosociaux auprès des enfants en "rupture du lien familial"}

Tout au long de cette présentation, nous voulions souligner le fait que l'absence d'une structure familiale contenante, d'origine ou substitutive, rend les liens d'attachement et les fonctions sociales plus fragiles, vulnérables. C'est bien le vécu de Soufian.

Face aux enfants abandonnés ou vivant des ruptures familiales définitives, les intervenants psychosociaux gagnèrent à mieux comprendre le type d'attachement et les modalités de socialisation qui les caractérise, sans oublier pour autant que leur compétence professionnelle ne remplace pas leur engagement personnel essentiel dans ce type de prise en charge.

L'enfant abandonné a besoin de reconstituer des liens affectifs de qualité - source de résilience -, au sein d'une structure sociale, familiale ou substitutive stable. C'est dans le miroir de l'autre, d'un autre significatif que se construit l'enfant. Il suffit donc de la présence d'un autre capable de devenir modèle identitaire pour que l'enfant dépasse, pas toujours, ses manques existentiels.

Bien entendu, chaque culture propose de modèles substitutifs de parenté pour les enfants orphelins, soit parce qu'un aîné de famille reprend la responsabilité de ces enfants, ou parce que dans la famille élargie on trouve une structure substitutive. Mais évidemment, l'affaiblissement généralisé du lien social rend problématique cette histoire naturelle de l'enfance abandonnée. C'est précisément à cause de ce manque, que le drame humain devient un fait psychopathologique et se crée un système institutionnel, dont les avatars sont bien connus. Nul doute qu'une société gouvernée par des principes de justice, de solidarité, de vérité et de liberté, une société fondée sur la paix, pourrait diminuer radicalement le nombre d'enfants sans famille et contribuer à gérer plus sereinement les situations de rupture inévitables.

Certes, les ruptures du lien familial peuvent bénéficier des mesures de prévention. Dès lors, les personnes concernées par le bien-être de l'enfant sont invitées à réfléchir sur les façons d'intervenir, avec honnêteté et à propos, soit pour prévenir les situations d'abandon ou de rupture, soit pour accueillir les enfants en souffrance. Il s'agit de soutenir l'humain, et en particulier "l'intérêt supérieur de l'enfant".

Dans le domaine de la maltraitance, par exemple, certaines études américaines montrent le bienfait d'encadrer des jeunes mères célibataires par un accompagnement social adéquat avant et après l'accouchement. Encore faut-il être conscients de toute l'ambiguïté que comporte une politique de prévention.

Le travail en réseaux communautaire est probablement rentable dans tous les cas, parce qu'il s'agit de mobiliser les ressources de la communauté et de promouvoir l'empowerment des citoyens'. Nous pensons à tout le travail qui se fait, par exemple, en Amérique Latine, avec les enfants de la rue, les enfants privés de liberté, etc. Nous pensons aussi aux programmes de professionnalisation mis en place en France pour approcher le problème des mineurs étrangers, pour la plupart des roumains, non accompagnés. Dans ce programme, outre des institutions de formation - par exemple, les Apprentis Orphelins d'Auteil, interviennent aussi des organismes officiels tant français que roumains.

En ce qui concerne des interventions plus précisément thérapeutiques, l'histoire de vie utilisée avec des enfants adoptés, perturbés socialement peut se montrer très utile. La reconstitution d'un fil conducteur de leur existence, à travers d'albums photographiques, des morceaux de souvenirs, propres ou venant de leur contexte, sont des éléments de réconciliation du sujet avec son histoire personnelle et avec la transmission générationnelle. C'est en s'appuyant sur son passé, sur la recherche de solides attaches du passé, que le sujet peut embrasser l'avenir. Or, l'enfant abandonné, privé de milieu familial, n'a pas réussi à établir ses liens de base suffisamment solides dans son propre passé. Il est contraint à les reconstituer ou les récréer. D'autre part la valeur des contes peut aussi faciliter la restructuration psychologique de ces enfants. Nous cherchons ainsi à reconstituer, ne fusse que symboliquement, une certaine forme d'ancrage familial.

Le travail thérapeutique institutionnel suppose une prise de conscience claire du type d'attachement manifesté par l'enfant. Il est donc important de favoriser le développement des fonctions sociales: proposer des jeux et activités de coopération (d'équipe); utiliser des techniques de développement de l'estime de soi, de l'assertivité, de la responsabilité; encourager les motivations et la résilience; proposer une éducation centrée sur les valeurs (philosophie avec les enfants) etc. Ce travail permet de rétablir des repères symboliques fonctionnels et structurants.

Donner la parole à l'enfant, quand cela est possible, et responsabiliser les enfants plus âgés à l'égard des plus jeunes (child-to-child), sont essentiels pour le travail thérapeutique. D'autre part, il est importante de prendre conscience de la provocation sous-jacente que l'enfant dyssocial utilise dans ses échanges avec les autres. C'est à l'adulte d'assumer des attitudes positives et structurantes pour ne pas se laisser embarquer dans des relations symétriques interminables qui apportent peu à la fonction contenante indispensable pour redresser "la croissance" de ces enfants.

\section{Réflexion finale...}

Nous nous étions proposés d'analyser l'impact de l'affaiblissement ou des "ruptures" du lien familial dans l'émergence des difficultés psychopathologiques. Pour réaliser ce projet nous sommes partis de l'hypothèse que l'absence de structure familiale contenante fournissant à l'enfant des repères symboliques, pouvait se traduire par l'émergence de comportements dysfonctionnels. 
Certes, le chantier est vaste et complexe, ce qui nous a poussé à nous limiter à décrire l'attachement et ses avatars comme un élément fondamental pour la compréhension de ces "ruptures" du lien social.

Il est nécessaire que les enfants en rupture du lien social récréent et/ou rétablissent des formes d'attachement (de relation) empathiques, contenantes et référentielles pour sortir de la spirale du risque de dysocialisation. $\mathrm{Au}$ moment de conclure, nous aimerions souligner que la famille constitue, de notre point de vue, le lieu privilégié de l'avènement de l'autre.

Dans bien des situations, il s'agit de les aider à entreprendre un voyage de reconstruction de soi: la vie a peut-être mal commencé, mais elle peut mieux avancer, en permettant à tout le moins qu'une autre histoire commence, grâce à la reconstitution des liens familiaux ou substitutifs contenants et stables.

\section{REFERÊNCIAS}

Boris, N. W.; Zeanah, C. R.; Larrieu, J. A.; Scheering, M. S. \& Heller, S. S. (1998). Attachment disorder in infancy and early childhood: a preliminary investigation of diagnostic criteria. American Journal of Psychiatry, 155, 295-297.

Bowlby, J. (1969). Attachement et perte. Vol . 1 : L'attachement. Vol. 2: Séparation, angoisse et colère. Vol. 3: La perte, tristesse et séparation. Paris : PUF.

Chisholm, K. (1998). A three-year follow-up of attachment and indiscriminate friendliness in children adopted from Romanian orphanages. Child Development, 69, 1092-1106.

Défenseur des Enfants de France (2001). Rapport Annuel. Défenseur des Enfants de France.

Goldfarb, W. (1945). Psychological privation in infancy and subsequent adjustment. American Journal of Orthopsychiatry, $14,247-255$

Lieberman, A. F. (1991). Attachment theory and infant-parent psychotherapy some conceptual, clinical and research consideration. Em D. Cicchettiu \& S. Toth (Orgs.), Rochester Symposium on development psychopathology. Vol. 3: Models and Integration (pp. 261-287). Rochester : University of Rochester Press.

Lieberman, A. F. \& Zeanah, C. R. (1995). Disorders of attachment. Child and Adolescent Psychiatry Clinics of North America, 4, 571-588.

Provence, S. \& Lipton, R. C. (1962). Infants reared in institutions. New York : International University Press.

O'Connor, T. G.; Bredenkamp, D., Rutter, M. \& the English and Romanian Adoption Adopters Study Team (1999). Attachment disturbances and disorders in children exposed to early severe deprivation. Infant Mental Health Journal, 120, 10-29.

O'Connor, T. G. (2000). Attachment disorder behavior following early severe deprivation: extension and longitudinal follow-up. Journal of the American Academy of Child and Adolescent Psychiatry, 39, 6, 703-712.

Rutter, M. \& O'Connor, T. G. (1999) Implications of attachment theory for childcare policies. Em $J$. Cassidy \& P. Shaver (Orgs.), Handbook of attachment (pp. 823-844). New York: Guildford.

Serrano, J. A. (2002). L'impact psychologique du divorce sur les enfants. Familia et Vita, VII (3), 26 - 41.

Serrano-Bascopé, A. M. (2003). Propositions for an ethical education program. Document de travail pour le GNRC Europe.

Tizard, B. \& Rees, J. (1975). The effect of early institutional rearing on the behavioural problems and affectional relationships of four-year-old children. Journal of Clinical Psycho- logy and Psychiatry, 16, 61-73.

Zeanah, C. R. (1996). Beyond insecurity: a reconceptualization of attachment disorders of infancy. Journal of Consulting and Clinical Psychology, 64, 42-52.

Wallerstein, J. S.; Lewis, J. M. \& Blakeslee, S. (2000). The unexpected legacy of divorce. New York: Hyperion.

Recebido: 14.10 .2003

Revisado: 30.11 .2003

Aceito: 10.12 .2003 


\begin{abstract}
Notas:
${ }^{1}$ Exigences imposées aux pays signataires de la Convention.

${ }^{2}$ Wallerstein, Lewis et Blakeslee (2000) dans un livre remarquable évoquent la souffrance vécue suite au divorce des parents et considèrent que la société américaine vit dans une véritable "culture du divorce", paradigme qui se répand largement en Europe occidentale.

${ }^{3}$ Cfr. Document de travail d'Ana María Serrano pour le GNRC - Europe, 2003.

${ }^{4}$ Qu'on le veuille ou non, le processus normatif demeure une exigence permanente de la famille, quand bien même les normes juridiques ou sociales qui la gouvernent sont, dans leur contenu, changeantes et contingentes (Encyclopédie Universalis).

${ }^{5}$ Il s'agit de sujets apparemment sociables, mais étrangement détachés, ils se montrent indifférents, sans affection, peu réactifs à la punition.

${ }^{6}$ Bowlby formulera les termes d' "exclusion défensive" des affects et de "confiance en soi compulsive" (compulsive selfreliance), terme traduit aussi comme "autonomie compulsive"). [Because a child with an attachment disorder has failed to make a secure attachment, he or she feels the need to be in control of the situation at all times].

${ }^{7}$ Processus d' "empowerment" l'empowerment est un processus par lequel une personne, qui se trouve dans des conditions de vie plus ou moins incapacitantes, développe, par I'intermédiaire d'actions concrètes, le sentiment qu'il est possible d'exercer un plus grand contrôle sur les aspects de sa réalité psychologique ou sociale qui sont importantes pour elle ou pour ses proches.
\end{abstract}

\title{
Sobre os autores:
}

Prof. Dr. Jorge Alberto Serrano: Pédopsychiatre. Professeur émérite. Faculté de Médecine. Université Catholique de Louvain. Cliniques Universitaires Saint Luc. Adresse pour la correspondance: Av. Hippocrate 10/2160 - 1200 Bruxelles/Belgique Courriel: serrano@nops.ucl.ac.be.

Lic. Verónica Serrano: Psychologue clinicienne. Chargée de cours. Faculté d'Humanités et des Sciences de l'Education. Universidad Mayor de San Simón. Cochabamba. Adresse pour la correspondance: Edificio Los Sauces $6^{\circ}$ B - Avenida América Oeste - Cochabamba/Bolivia - Correo electrónico: serran7@ hotmail.com. 